\title{
Transition to Instability in a Kicked Bose-Einstein Condensate
}

\author{
Chuanwei Zhang ${ }^{1,2}$, Jie Liu ${ }^{1,3}$, Mark G. Raizen ${ }^{1,2}$, and Qian Niu ${ }^{1}$ \\ ${ }^{1}$ Department of Physics, The University of Texas, Austin, Texas 78712-1081 \\ ${ }^{2}$ Center for Nonlinear Dynamics, The University of Texas, Austin, Texas 78712-1081 \\ ${ }^{3}$ Institute of Applied Physics and Computational Mathematics, P.O.Box 100088, Beijing, P. R. China
}

\begin{abstract}
A periodically kicked ring of a Bose-Einstein condensate is considered as a nonlinear generalization of the quantum kicked rotor. For weak interactions between atoms, periodic motion (anti-resonance) becomes quasiperiodic (quantum beating) but remains stable. There exists a critical strength of interactions beyond which quasiperiodic motion becomes chaotic, resulting in an instability of the condensate manifested by exponential growth in the number of noncondensed atoms. Similar behavior is observed for dynamically localized states (essentially quasiperiodic motions), where stability remains for weak interactions but is destroyed by strong interactions.
\end{abstract}

PACS numbers: 05.45.-a, 03.75.-b, 03.65.Bz, 42.50.Vk

The classical kicked rotor is a textbook paradigm for dynamical chaos 1]. The quantum kicked rotor has play an equally important role for the study of quantum chaos, for which a wide range of effects have been predicted 2] and observed in experiments 3 . In recent years, the realization of Bose-Einstein condensation (BEC) of dilute gases [4] has opened new opportunities for studying dynamical systems in the presence of many-body interactions. A natural question to ask is how the physics of the quantum kicked rotor is modified by interactions [5].

In the classical regime, chaotic motion leads to diffusive growth in the kinetic energy. In quantum dynamics, where chaos is no longer possible because of the linearity of the Schrödinger equation, the motion becomes periodic (anti-resonance), quasiperiodic (dynamical localization), or resonant (quantum resonance) 6, 7]. In the mean field approximation, many-body interactions in BEC are represented by adding a nonlinear term in the Schrödinger equation [8]. This nonlinearity makes it possible to bring chaos back into the system, leading to instability (in the sense of exponential sensitivity to initial conditions) of the condensate wave function [9]. The onset of instability of the condensate can cause rapid proliferation of thermal particles [10] that can be observed in experiments 11]. It is therefore important to understand the route to chaos with increasing interactions. This problem has recently been studied for the kicked BEC in a harmonic oscillator [12].

In this Letter, we investigate the quantum dynamics of a BEC with repulsive interaction that is confined on a ring and kicked periodically. This system is a nonlinear generalization of the quantum kicked rotor. From the point of view of dynamical theory, the kicked rotor is more generic than the kicked harmonic oscillator, because it is a typical low dimensional system that obeys the KAM theorem [13]. It is very interesting to understand how both quantum mechanics and mean field interaction affect the dynamics of such a generic system. We will focus most of our attention on the case of anti-resonance because it is the simplest periodic mo- tion. Here we find, with both analytic and numerical calculations, that weak interactions will make the motion quasiperiodic in the form of quantum beating. For strong interactions, quasiperiodic motions are destroyed and we observe a transition to instability of the BEC that is also characterized by an exponential growth in the number of noncondensed atoms. Universal critical behavior for the transition is found. We have also studied nonlinear effect on dynamically localized states that may be regarded as quasiperiodic. Similar results are obtained in that localization remians for sufficiently weak interactions but become unstable beyond a critical strength of interactions.

Consider condensed atoms confined in a toroidal trap of radius $R$ and thickness $r$, where $r \ll R$ so that lateral motion is negligible and the system is essentially one-dimensional 14]. The dynamics of the BEC is described by the dimensionless nonlinear Gross-Pitaveskii (GP) equation,

$$
i \frac{\partial}{\partial t} \psi=\left(-\frac{1}{2} \frac{\partial^{2}}{\partial \theta^{2}}+g|\psi|^{2}+K \cos \theta \delta_{t}(T)\right) \psi,
$$

where $g=8 N a R / r^{2}$ is the scaled strength of nonlinear interaction, $N$ is the number of atoms, $a$ is the $s$-wave scattering length, $K$ is the kick strength, $\delta_{t}(T)$ represents $\sum_{n} \delta(t-n T), T$ is the kick period, and $\theta$ denotes the azimuthal angle. The length and the energy are measured in units $R$ and $\frac{\hbar^{2}}{m R^{2}}$, respectively. The wavefunction $\psi(\theta, t)$ has the normalization $\int_{0}^{2 \pi}|\psi|^{2} d \theta=1$ and satisfies periodic boundary condition $\psi(\theta, t)=\psi(\theta+2 \pi, t)$. Experimentally, the ring-shape potential may be realized using two 2D circular "optical billiards" with the lateral dimension being confined by two plane optical billiards 15]. The $\delta$-kick may be realized by adding potential points along the ring with an off-resonant laser [3]. The interaction strength $g$ may be adjusted using a magnetic field-dependent Feshbach resonance [16].

The mean energy of each particle is $E(t)=$ $\int_{0}^{2 \pi} d \theta \psi^{*}\left(-\frac{1}{2} \frac{\partial^{2}}{\partial \theta^{2}}+\frac{1}{2} g|\psi|^{2}\right) \psi$. To determine the evolu- 


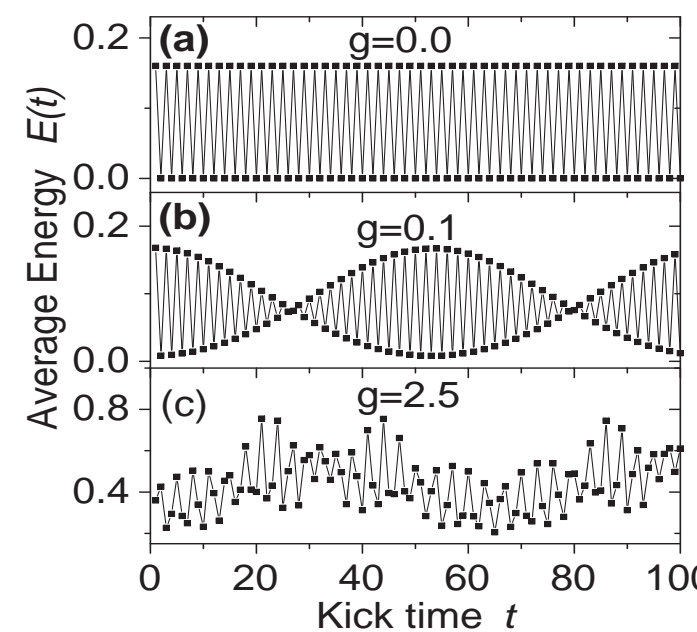

Figure 1: Plots of average energy $E(t)$ versus the number of kicks $t$ for three values of $g$. The kick strength $K=0.8$.

tion of the energy, we numerically integrate Eq.(1) over a time span of 100 kicks, using a split-operator method [17], with the initial wavefunction $\psi$ being the ground state $\psi(\theta, 0)=1 / \sqrt{2 \pi}$. The kick period is chosen as $T=2 \pi$ to match the condition for anti-resonance. After each kick, the energy $E(t)$ is calculated and plotted as shown in Fig.1. We see a remarkable difference among noninteraction (Fig.1(a)), weak interaction (Fig.1(b)), and strong interaction (Fig.1(c)) cases. For noninteraction, the energy $E(t)$ oscillates between two values (anti-resonance) and the period is $2 T$; While in the weak interaction case, the amplitude of the oscillation decreases gradually to zero and then revives, similar to the phenomena of beating in classical waves. The values of the oscillation and beat frequencies are obtained by Fourier Transform and the results are presented in Fig.2. It is shown that, besides the appearance of the beat frequency, the interaction also modifies the oscillation frequency; For stronger interaction (Fig.1(c)), i.e. $g \geq 1.96$, we find that the energy's evolution demonstrates an irregular pattern, clearly indicating the collapse of the quasiperiodic motion and the occurrence of instability.

For the case of weak interaction, the system is pre-

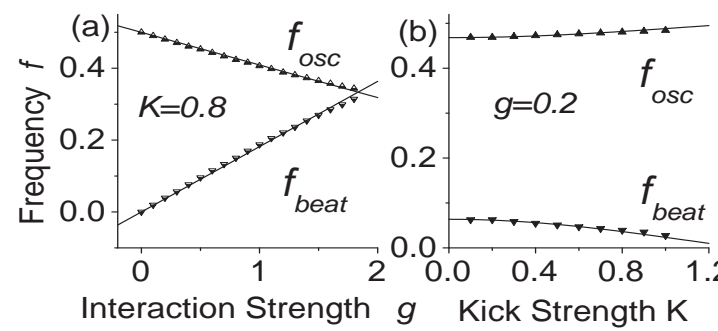

Figure 2: Plots of beat and oscillation frequencies versus the interaction strength (a) and kick strength (b), where the scatters are the results from numerical simulation using GP equation and lines from analytic expression Eq.(3). dominantly in the two energy levels. We can map the system onto a spin model with a two-mode approximation to the GP equation 18]. By considering the conservation of parity we may write the wavefunction as $\psi=\frac{1}{\sqrt{2 \pi}}(a+\sqrt{2} b \cos \theta)$, where the populations $a$ and $b$ at the ground and excited states satisfy the normalization condition $|a|^{2}+|b|^{2}=1$. The Hamiltonian in the spin representation reads

$$
\mathcal{H}=-\frac{S_{z}}{2}+\frac{g}{2 \pi}\left(S_{x}^{2}-\frac{S_{z}}{4}+\frac{S_{z}^{2}}{8}\right)+\sqrt{2} K S_{x} \delta_{t}(T),
$$

where $S_{z}$ corresponds to the population difference $|a|^{2}-$ $|b|^{2}$ and $\arctan \left(S_{y} / S_{x}\right)$ gives the relative phase $\alpha=$ $\arg (a)-\arg (b)$. This Hamiltonian is similar to a kicked top model 19], but here the evolution between two kicks is more complicated.

With the spin model, we can readily study the dynamics of the system. For the case of noninteraction, the evolution between two consecutive kicks is simply an angle $\pi$ rotation about the $z$ axis. The spin initially directing to north pole stays there for time duration $T$, then the first kick rotates the spin by an angle $\sqrt{2} K$ about the $x$ axis. The following free evolution rotates the spin by an angle $\pi$ about the $z$ axis. Then, the second kick will drive the spin back to north pole through another rotation of $\sqrt{2} K$ about the $x$ axis. With this the spin's motion is two kick period recurrence and quantum anti-resonance occurs.

With interaction, the motion between two consecutive kicks is approximately described by a rotation of $\pi+g\left(1+3 S_{z}\right) / 4$ about the $z$ axis. Compared with the noninteraction case, the mean field interaction leads to an additional phase shift $g\left(1+3 S_{z}\right) / 4$. This phase shift results in a deviation of the spin from $S_{x}=0$ plane at time $2 T^{-}$, i.e., moment just before the second kick. As a result, the second kick cannot drive the spin back to its initial position and quantum anti-resonance is absent. However, the phase shift will be accumulated in future evolution and the spin may reach $S_{x}=0$ plane at a certain time $m T^{-}$(beat period) when the total accumulated phase shift is $\pi / 2$. Then the next kick will drive spin back north pole by applying an angle $\sqrt{2} K$ rotation about the $x$ axis.

The above picture is confirmed by our numerical solution of the spin Hamiltonian with fourth-order RungeKutta method [20]. In Fig.3 we see that, the relative phase at the moment just before the even kicks increases almost linearly and reaches $2 \pi$ in a beat period. The slope of the increment reads, $\gamma_{R P}=$ $\left(\alpha\left(4 T^{-}\right)-\alpha\left(2 T^{-}\right)\right) / 2$, which can be deduced analytically. With this and through a lengthy deduction, we obtain an analytic expression for the beat frequency to first order in $g$,

$$
f_{\text {beat }} \approx \frac{g}{4 \pi}(1+3 \cos (\sqrt{2} K)) .
$$



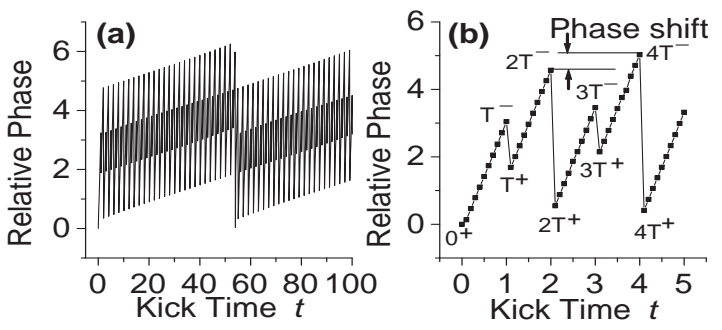

Figure 3: (a) Plots of relative phase versus the number of kicks $t$, where $g=0.1, K=0.8$. (b) Schematic plot of the phase shift. $n T^{-(+)}$represents the moment just before (after) the $n$th kick.

This expression as well as the relation between the oscillation frequency and the beat frequency, $f_{\text {osc }}=\frac{1}{2}-\frac{1}{2} f_{\text {beat }}$, is in very good agreement with the numerical results as shown in Fig.2. Therefore the beating provides a method to measure interaction strength in an experiment.

Tuning the interaction strength still larger means enhancing further the nonlinearity of the system. From our general understanding of nonlinear systems, we expect that the solution will be driven towards chaos, in the sense of exponential sensitivity to initial condition and random evolution in the temporal domain. The latter character has been clearly displayed by the irregular pattern of the energy evolution in Fig.1(c). On the other hand, the onset of instability (or chaotic motion) of the condensate is accompanied with the rapid proliferation of thermal particles. Within the formalism of Castin and Dum [10], the growth of the number of the noncondensed atom will be exponential, similar to the exponential divergence of nearby trajectories in phase space of classical system. The growth rate of the noncondensed atoms is similar to the Lyapounov exponent, turning from zero to nonzero as instability occurs.

In Castin and Dum's formalism, the mean number of noncondensed atoms at zero temperature is described by $\langle\delta \hat{N}(t)\rangle=\sum_{k=1}^{\infty}\left\langle v_{k}(t) \mid v_{k}(t)\right\rangle$, where $\left|v_{k}(t)\right\rangle$ are governed by

$i \frac{d}{d t}\left(\begin{array}{c}u_{k} \\ v_{k}\end{array}\right)=\left(\begin{array}{cc}H+g Q|\psi|^{2} Q & g Q \psi^{2} Q^{*} \\ -g Q^{*} \psi^{* 2} Q & -H-g Q^{*}|\psi|^{2} Q^{*}\end{array}\right)\left(\begin{array}{c}u_{k} \\ v_{k}\end{array}\right)$,

where $H=\frac{\hat{p}^{2}}{2}+g|\psi|^{2}-\mu, \mu$ is the chemical potential, $\psi$ is the ground state of GP equation and the projection operators $Q$ are given by $Q=1-|\psi\rangle\langle\psi|$.

We numerically integrate Eq.(4) for the $u_{k}, v_{k}$ pairs over a time span of 100 kicks, using a split operator method, parallel to numerical integration of GP equation. The initial conditions $\left|u_{k}(0)\right\rangle,\left|v_{k}(0)\right\rangle$, for initial ground state wavefunction $\psi(\theta)=1 / \sqrt{2 \pi}$, are obtained by diagonalizing the linear operator in Eq.(4) [21]. After each kick the mean number of noncondensed atoms is calculated and plotted versus time in Fig.4(a). We find that there exists a critical value for the interaction
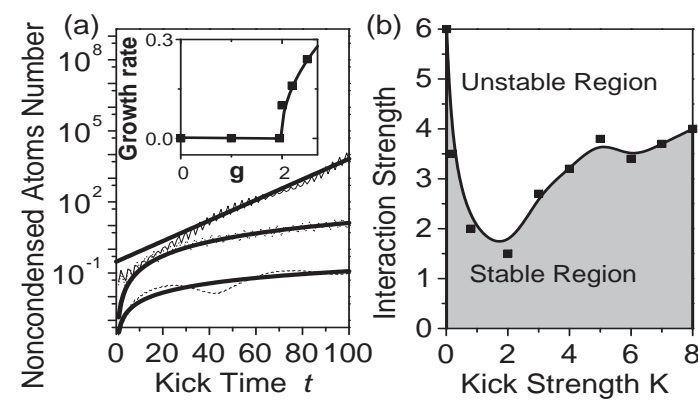

Figure 4: (a) Semilog plot of the mean number of noncondensed atoms versus the number of kicks $t$. The thicker lines are fitting functions. $K=0.8, g=0.1$ (dashed line, fitting function $\left.0.0003 t^{1.3}\right), g=1.5$ (dotted line, fitting function $\left.0.0011 t^{2}\right), g=2.0$ (dash dotted line, fitting function $0.32 \exp (0.1 \mathrm{t}))$. The inset shows the interaction dependence of the growth rate. The scatters are from numerical simulation and the solid line is the fitting function $0.33(g-1.96)^{1 / 2}$. (b) Phase diagram of the transition to instability.

strength, i.e., $g_{c}=1.96$, above which, the mean number of noncondensed atoms increases exponentially, indicating the instability of BEC. Below the critical point, the mean number of noncondensed atoms increases polynomially. As the nonlinear parameter crosses over the critical point, the growth rate turns from zero to nonzero, following a square-root law (inset in Fig.4(a)). This scaling law may be universal for Bogoliubov excitation as confirmed by recent experiments [1].

The critical value of the interaction strength depends on the kick strength. For very small kick strength, the critical interaction is expected to be large, because the ground state of the ring-shape BEC with repulsive interaction is dynamically stable 22]. For large kick strength, to induce chaos, the interaction strength must be large enough to compete with the external kick potential. So, in the parameter plane of $(g, k)$, the boundary of instability shows a "U" type curve (Fig.4(b)).

Across the critical point, the density profiles of both condensed and noncondensed atoms change dramatically. In Fig.5, we plot the temporal evolution of the density distributions of condensed atoms as well as noncondensed atoms. In the stable regime, the condensate density oscillates regularly with time and shows clear beating pattern (Fig.5(a)), whereas the density of the noncondensed atoms grows slowly and shows main peaks around $\theta= \pm \pi$ and 0 , besides some small oscillations (Fig.5(b)). In the unstable regime, the temporal oscillation of the condensate density is irregular (Fig.5(c)), whereas the density of noncondensed atoms grows explosively with the main concentration peaks at $\theta= \pm \pi / 2$ where the gradient density of the condensed part is maximum (Fig.5(d)). Moreover, our numerical explorations show that the $\cos ^{2} \theta$ mode (Fig5.(b)) dominates the density distribution of the noncondensed atoms as the interaction strength is less than 1.8. Thereafter, the $\sin ^{2} \theta$ mode grows while $\cos ^{2} \theta$ 

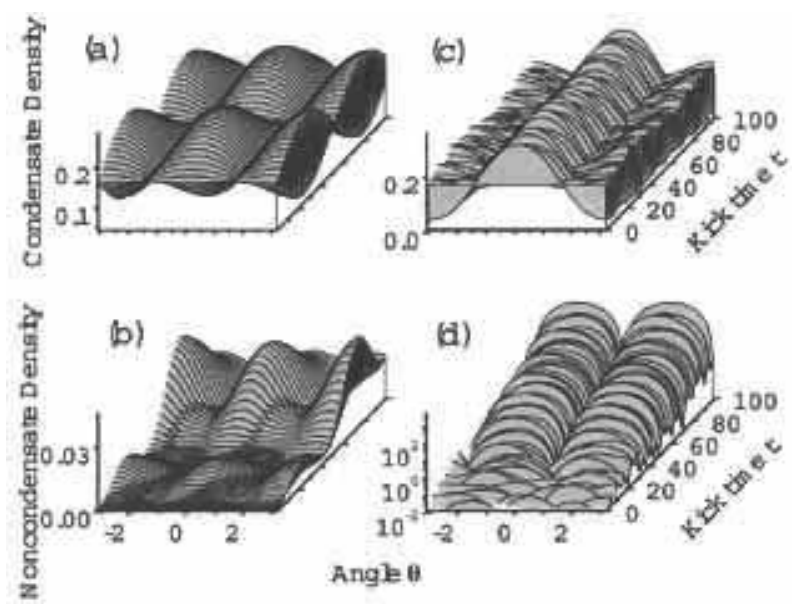

Figure 5: Plots of condensate and noncondensate densities, where $K=0.8$. (a,b) $g=0.1$; (c,d) $g=2.0$.

mode decays, and finally $\sin ^{2} \theta$ mode become dominating in the density distribution of noncondensed atoms above the transition point (Fig5.(d)). Since the density distribution can be measured in experiment, this effect can be used to identify the transition to instability.

Finally, although the above discussions have been focused on a periodic state of anti-resonance, the transition to instability due to strong interactions also follows a similar path for a dynamically localized state 23. The only difference is that we start out with a quasiperiodic rather than periodic motion in the absence of interaction. This means that it will generally be easier to induce instability but still requires a finite strength of interaction. In Fig.6, we show the nonlinear effect on a dynamically localized state at $K=5$ and $T=1$. For weak interactions $(g=1)$ the motion is quasiperiodic with slow growth in the number of noncondensed atoms. Strong interaction $(g=5)$ destroys the quasiperiodic motion and leads to diffusive growth of energy, accompanied with exponential growth of noncondensed atoms that clearly indicates the instability of the BEC. Notice that the rate of growth in energy is much slower than the classical diffusion rate, which means that chaos brought back by interaction in

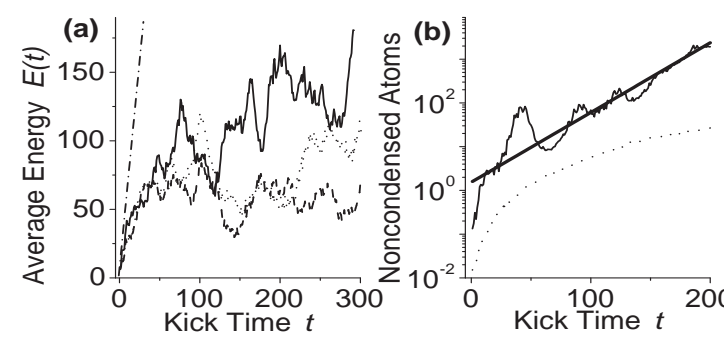

Figure 6: Nonlinear effects on dynamically localized states. $K=5, T=1$. (a) Plots of average energy $E(t)$ versus the number of kicks $t$, where dash dotted line corresponds to the classical diffusion. $g=0$ (dash), $g=1$ (dot), $g=5$ (solid). (b) Semilog plot of the mean number of noncondensed atoms versus the number of kicks $t . g=1$ (dot), $g=5$ (solid). this quantum system is still much weaker than pure classical chaos.

We acknowledge the support from the NSF, and the R. A. Welch foundation, MGR also acknowledges supports from the Sid W. Richardson Foundation.

[1] L. E. Reichl, The Transition to Chaos In Conservative Classical Systems: Quantum Manifestations (SpringerVerlag, New York 1992) and references therein .

[2] G. Casati, et al., Lect. Notes Phys. 93, 334 (1979); B.V. Chirikov, Phys. Rep. 52, 263 (1979); F.M. Izrailev, ibid 196, 299 (1990).

[3] F.L. Moore, et al., Phys. Rev. Lett. 75, 4598 (1995); H. Ammann, et al., ibid 80, 4111 (1998); M.B. d'Arcy, et al. ibid, 87, 074102 (2001); W.H. Oskay, et al., Opt. Comm. 179, 137 (2000).

[4] M.H. Anderson, et al., Science 269, 198 (1995); K.B. Davis, et al., Phys. Rev. Lett. 75, 3969 (1995); C.C. Bradley, et al., ibid 79, 1170 (1997).

[5] F. Benvenuto, et al., Phys. Rev. A 44, R3423 (1991); D.L. Shepelyansky, Phys. Rev. Lett. 70, 1787 (1993).

[6] T. Hogg and B.A. Huberman, Phys. Rev. Lett. 48, 711 (1982); S. Fishman, et al., ibid 49, 509 (1982).

[7] F.M. Izrailev, and D.L. Shepelyansky, Theor. Math. Phys. 43, 553 (1980); I. Dana, et al., Phys. Rev. Lett. 74, 686 (1995).

[8] F. Dalfovo, et al., Rev. Mod. Phys. 71, 463 (1999); A.J. Leggett, ibid 73, 307 (1999).

[9] A. Vardi and J.R. Anglin, Phys. Rev. Lett. 86, 568 (2001); G.P. Berman, et al., ibid 88, 120402 (2002).

[10] Y. Castin and R. Dum, Phys. Rev. A 57, 3008 (1998); Phys. Rev. Lett. 79, 3553 (1997).

[11] J.K. Chin, et al., Phys. Rev. Lett. 90, 160405 (2003).

[12] S.A. Gardiner, et al., Phys. Rev. A 62, 023612 (2000); R. Artuso and L. Rebuzzini, Phys. Rev. E 66, 017203 (2002).

[13] B. Hu, et al., Phys. Rev. E 58, 1743 (1998); B. Hu, et al., Phys. Rev. Lett. 82, 4224 (1999).

[14] L.D. Carr, et al., Phys. Rev. A 62, 063610 (2000); G.M. Kavoulakis, ibid 67, 011601 (2003); R. Kanamoto, et al., ibid 67, 013608 (2003).

[15] V. Milner,et al., Phys. Rev. Lett. 86, 1514 (2001); N. Friedman, et al., ibid 86, 1518 (2001).

[16] J.M. Gerton, et al., Nature 408, 692 (2000); E.A. Donley, et al., ibid 412, 295 (2001).

[17] A.D. Bandrauk, and H. Shen, J. Phys. A 27, 7147 (1994).

[18] J. Liu, et al., Phys. Rev. Lett. 90, 170404 (2003); J. Liu, et al., Phys. Rev. A 66, 023404 (2002).

[19] F. Haake, Quantum Signature of Chaos (Springer-Verlag, New York 2000).

[20] W.H. Press, et al., Numerical Recipes in C++ (Cambridge, New York 2000).

[21] Y. Castin, in Coherent Atomic Matter Waves, edited by R. Kaiser, et al., (EDP Sciences and Springer-Verlag 2001).

[22] B. Wu and Q. Niu, Phys. Rev. A 64, 061603 (2001); J.R. Anglin, ibid 67, R051601 (2003).

[23] More details will be provided in the future publication. 